\title{
A Joint Channel Selection and Routing Protocol for Cognitive Radio Network
}

\author{
Yuting Wang $\mathbb{D},{ }^{1}$ Guoqiang Zheng, ${ }^{1}$ Huahong Ma $\mathbb{D}^{1},{ }^{1}$ Yang Li, ${ }^{1}$ and Jishun Li $\mathbb{D}^{2}$ \\ ${ }^{1}$ School of Information Engineering, Henan University of Science and Technology, Luoyang 471023, China \\ ${ }^{2}$ Henan Key Laboratory for Machinery Design and Transmission System, Luoyang 471023, China \\ Correspondence should be addressed to Yuting Wang; 18238801672@163.com
}

Received 10 August 2017; Revised 11 December 2017; Accepted 26 December 2017; Published 13 March 2018

Academic Editor: Bernard Cousin

Copyright (c) 2018 Yuting Wang et al. This is an open access article distributed under the Creative Commons Attribution License, which permits unrestricted use, distribution, and reproduction in any medium, provided the original work is properly cited.

\begin{abstract}
In cognitive radio network, the activities of primary users will cause great influence on the stability of multiple hops routes between cognitive users. In this regard, a joint channel selection and routing protocol, termed as CSRP, is proposed to ensure route stability and reduce route latency between cognitive users. The channel availability based on historical information and the channel switching delay are used as the channel selection criteria to choose the end-to-end shortest route which possesses high data delivery probabilities and low delays. Besides, simulation results show that the proposed protocol has a better performance in terms of packet transmission delay and data delivery rate compared with the routing protocols based on delay (TDRP) and based on joint routing and channel allocation (PUB-JRCA).
\end{abstract}

\section{Introduction}

The wireless communication technology since 20th century has been developed rapidly and has brought great convenience for people's life. Simultaneously, wireless communication technology is widely used in military, industrial, commercial, and many other fields. However, the existing limited spectrum resources are difficult to meet the current human communication needs. In order to alleviate the increasingly tense spectrum resources, cognitive radio technology, widely concerned, is a promising solution to solve the problem of spectrum scarcity and low spectrum utilization associated with classical fixed spectrum assignment schemes $[1,2]$. The core idea is that cognitive user using a unique detection device automatically detects the spectrum state on the current environment to find the idle channel and, without interfering with primary users, choose an opportunity to access the channel to achieve the purpose of spectrum multiplexing, improving network transmission performance [3]. The biggest difference between cognitive radio networks and traditional wireless networks is the application of cognitive radio technology, and the existing routing protocol is no longer applicable to this new type of network, so the original protocols need to be improved.
In terms of the problem of routing in cognitive environment, some algorithms and protocols have been put forward by domestic and foreign scholars. The OPERA [4] analyzes the optimal delay metric from the perspective of probability and the influence of the activity of primary users on the end-to-end delay. In [5], a SMART scheme is proposed that enables SUs to form clusters in a cognitive radio network and enables each SU source node to search for a route to its destination node on the clustered network. Based on the network conditions, SMART enables SUs to adjust their cluster size, which represents the number of nodes in a cluster, and searches for a route on the clustered network using an artificial intelligence approach called reinforcement learning. In [6], LAUNCH is presented as a location-aided routing protocol for CRNs that has a minimal route setup delay, prefers stable routes, handles primary users heterogeneity, and handles secondary users mobility. LAUNCH is based on four main concepts: a novel location aware CRN routing metric that takes into account the PUs activity; distributed calculations at the neighbors; a channel locking mechanism to achieve the route stability and minimize channel switching time; an efficient route maintenance strategy. In [7], a primary-user behavior based joint routing and channel allocation (PUB-JRCA) algorithm was proposed. According to 
the route discovery mechanism in the dynamic source routing protocol, the destination node selected the route which has the largest channel available probability. A node analytical model is proposed to describe the scheduling-based channel assignment progress in [8], which relieves the interflow interference and frequent switching delay. Obviously, stability and end-to-end delay are two important factors in the study of cognitive network routing [9]. Therefore, we present a joint interaction between channel selection and routing protocol, CSRP, taking into account availability and switching delay to select the channel with the shortest end-to-end delay as the best, improving the delay performance of route and the delivery rate of data.

The remainder of the paper is organized as follows. The opportunistic cognitive routing protocol is analyzed in Section 2. The performance evaluation is presented in Section 3. Concluding remarks are given in Section 4.

\section{Routing Protocol}

In this section, the new protocol CSRP is described in detail. Firstly, the problems of cognitive network are introduced. Then the network model and the channel selection algorithm are given. Finally, the four stages of routing protocol are analyzed.

2.1. Problem Description. The cognitive radio network is a secondary network, that is to say, only when the authorized band of first-level user is idle, these bands can be used by subusers which are also called cognitive nodes for data communication. So the channel selection algorithm must be considered when routing protocol is designed. In order to meet network performance requirements, each cognitive node in cognitive radio network must be able to switch between multiple available channels and select an appropriate idle channel for communication. Once cognitive user detects that primary user is using the channel, it must immediately and unconditionally withdraw. Therefore, channel switching delay resulting from channel frequently changing by cognitive nodes must be taken into account, and, especially in multihop network data transmission process, this problem is more obvious. We can explain the problem in Figure 1: there are two paths, namely, A-B-C and D-B-E. The channel number selected by $A$ and $C$ is 1 and channel 2 is selected by $\mathrm{D}$ and $\mathrm{E}$. In order to ensure the two paths communicate normally, node B should switch between channel 1 and 2 frequently, which results in switching delay.

On the other hand, the end-to-end stability is seriously affected by the activities of primary user in multihop routing, so the stability of each link must be taken into account. For example, there is a path S-a-b-D, and if the channel between $a$ and $b$ is not available due to the activity of primary user, the link will fail, and at this time whether the other link is connected or not, the whole link is not available. We cannot complete data transmission unless a route is rebuilt. However rebuilding routes will result in additional delay and more energy waste. So for multihop routing, the stability of route must be considered.

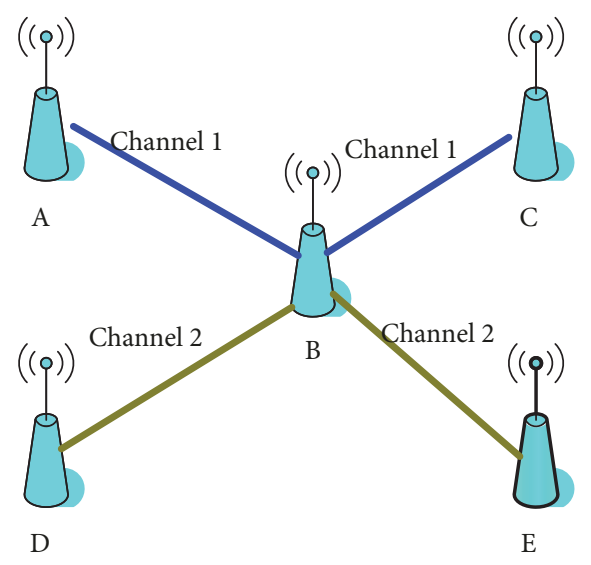

FIGURE 1: Channel switching frequently.

We propose a novel routing protocol CSRP with channel selection algorithm for cognitive radio network and, in the premise of ensuring routing stability, channel availability and switching delay are used as the selection criteria. In particular, the CSRP does not need central control node to manage the spectrum distribution information of the whole network and uses the channel availability based on the historical information to evaluate the usage of authorization channel, effectively reducing the interference of primary users in data transmission process and obtaining higher data delivery rates and lower data delivery delays.

2.2. System Model. Assume that there are $N$ cognitive users (SU) and $M$ primary user (PU) authorized users in cognitive radio network, and the following are the assumptions we made:

(1) Each authorized user owns an authorized channel and can communicate with each other through the authorized channel. There is a common control channel (CCC) and $M$ data channel (DC). Each cognitive user can transfer control message through CCC and there are a certain frequency intervals in the adjacent DC frequency band

(2) All cognitive users have the same transmission range and interference range and can obtain a set of available channel sets by spectrum sensing technology.

(3) For the link $(i, j)$ between node $i$ and $j, P_{i j}^{k}$ represents the probability of successfully transmitting data on channel $k$; that is, the link will not be disturbed by the authorized user.

(4) If $A(i)$ represents the available channel set (ACS) information of node $i$ and $A(j)$ represents the ACS information of node $j, A(i) \cap A(j)$ represents all available channels between nodes $i$ and $j$.

In Figure 2, PU1, PU2, and PU3 represent primary users. And $\mathrm{C} 1, \mathrm{C} 2$, and $\mathrm{C} 3$ denote the authorized frequency bands to which primary user is assigned. The other nodes represent different cognitive users, where $S$ is the source node and 


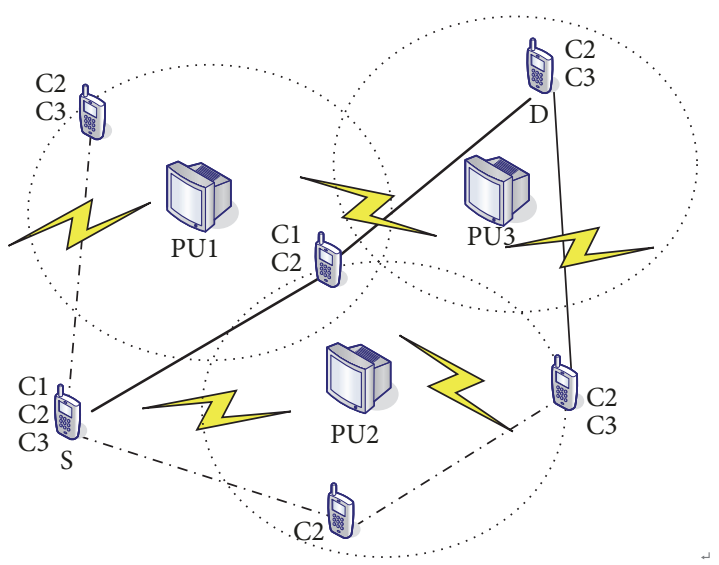

FIgURE 2: The cognitive radio network model.

$D$ is the destination node. And the available channel Set (ACS) marked nearby can be obtained by the cognitive user through spectrum sensing [10]. Normally, ACS are different for different cognitive users at the same time.

2.3. Channel Selection Algorithm. Neighboring nodes may have multiple available common channels in the communication range at the same time, so how to choose the optimal channel for data transmission becomes the key to ensuring routing performance. The CSRP takes channel availability probability and switching delay as the criterion of channel selection. Each cognitive node in network obtains its own available channel set ACS by diffusion spectrum sensing technology and simultaneously maintains an information table that records the available probability for each channel. In order to avoid periodic channel detection, the information table is based on the history information, which is expressed as $\left\langle P_{1}, P_{2}, P_{3}, \ldots, P_{i}, \ldots, P_{m}\right\rangle$, and $P_{i}$ represents the channel availability of node $i$ and $m$ represents the total number of channels. After cognitive node transmits data through channel $i$, the channel available probability is updated using the following manner:

$$
p_{i}= \begin{cases}p_{i}^{\prime}+\left(1-p_{i}^{\prime}\right) \cdot p_{\text {init }}, & \text { successful transmission } \\ p_{i}^{\prime} \cdot \gamma^{\mu}, & \text { others }\end{cases}
$$

where $p_{i}^{\prime}$ and $p_{i}$ represent the channel availability of node $i$. Because the channel is constantly updated, $p_{i}^{\prime}$ represents before the update and $p_{i}$ indicates after the update. $\gamma$ is channel availability update factor and is empirical value based on network traffic history information. Simultaneously, $\mu$ is the attenuation factor and is a positive integer.

Simultaneously, the channel switching delay resulting from switching channel frequently for data transmission cannot be ignored. And the channel switching delay is related to the number of frequencies of channel passing, which can be calculated from the following formula:

$$
S_{i}=f \times\left|B W_{\text {current }}-B W_{i}\right|,
$$

where $\left|B W_{\text {current }}-B W_{i}\right|$ represents the frequency interval from the current working channel to channel $i$ and $f$ is
TABLE 1: Channel state table of cognitive nodes.

\begin{tabular}{lcc}
\hline Channel serial number & Channel availability & $\begin{array}{c}\text { Channel switching } \\
\text { delay }\end{array}$ \\
\hline C1 & $P_{1}$ & $S_{1}$ \\
C2 & $P_{2}$ & $S_{2}$ \\
C3 & $P_{3}$ & $S_{3}$ \\
$\ldots \ldots$ & $\ldots \ldots$ & $\ldots \ldots$ \\
Cm & $P_{m}$ & $S_{m}$ \\
\hline
\end{tabular}

the scale factor. According to the statistics, in the $20 \mathrm{MHz}$ $30 \mathrm{GHz}$ spectral range, changing $10 \mathrm{MHz}$ will bring $10 \mathrm{~ms}$ switching delay [11], so $f$ is set to $10 \mathrm{~ms} / \mathrm{MHz}$.

So in the channel state information table of cognitive node, it is necessary to include the available probability of channel and the switching delay of shifting to this channel as shown in Table 1. The total number of channels is represented by $m$.

Finally, the cognitive node selects the best channel for data transmission according to its own channel state table. The process which node $y$ selects transmission channel after receiving the channel state table of node $x$ is shown as follows:

(1) Firstly, node $y$ selects the common available channel set of the two nodes according to the channel state table of the two nodes and extracts the channel availability probability and the switching delay.

(2) Node $y$ calculates the channel availability of each channel, respectively, according to

$$
P_{i}=P_{x i} \cdot P_{y i}
$$

where $P_{x i}, P_{y i}$ represent the available probability of node $x$ and node $y$ at channel $i$, respectively.

(3) Node $y$ calculates the channel switching delay for each channel, respectively, according to

$$
S_{i}=S_{x i}+S_{y i}
$$

where $S_{x i}, S_{y i}$ represent the delay cost of node $x$ and node $y$ switching to channel $i$, respectively.

(4) The weights of each eligible channel are calculated via

$$
\text { weight }=\alpha P_{i}-(1-\alpha) S_{i}
$$

and arranged in order from large to small. And the channel with the largest weight is selected as the data transmission. $\alpha$ represents a factor between 0 and 1 and is experience value selected based on network history information, and here we assume $\alpha=0.6$.

2.4. Protocol Implementation. The protocol we proposed is based on AODV routing mechanism and combines the channel selection algorithm for routing choice. The protocol implementation is divided into three phases: the route discovery phase, the route establishment phase, and the route maintenance phase. 


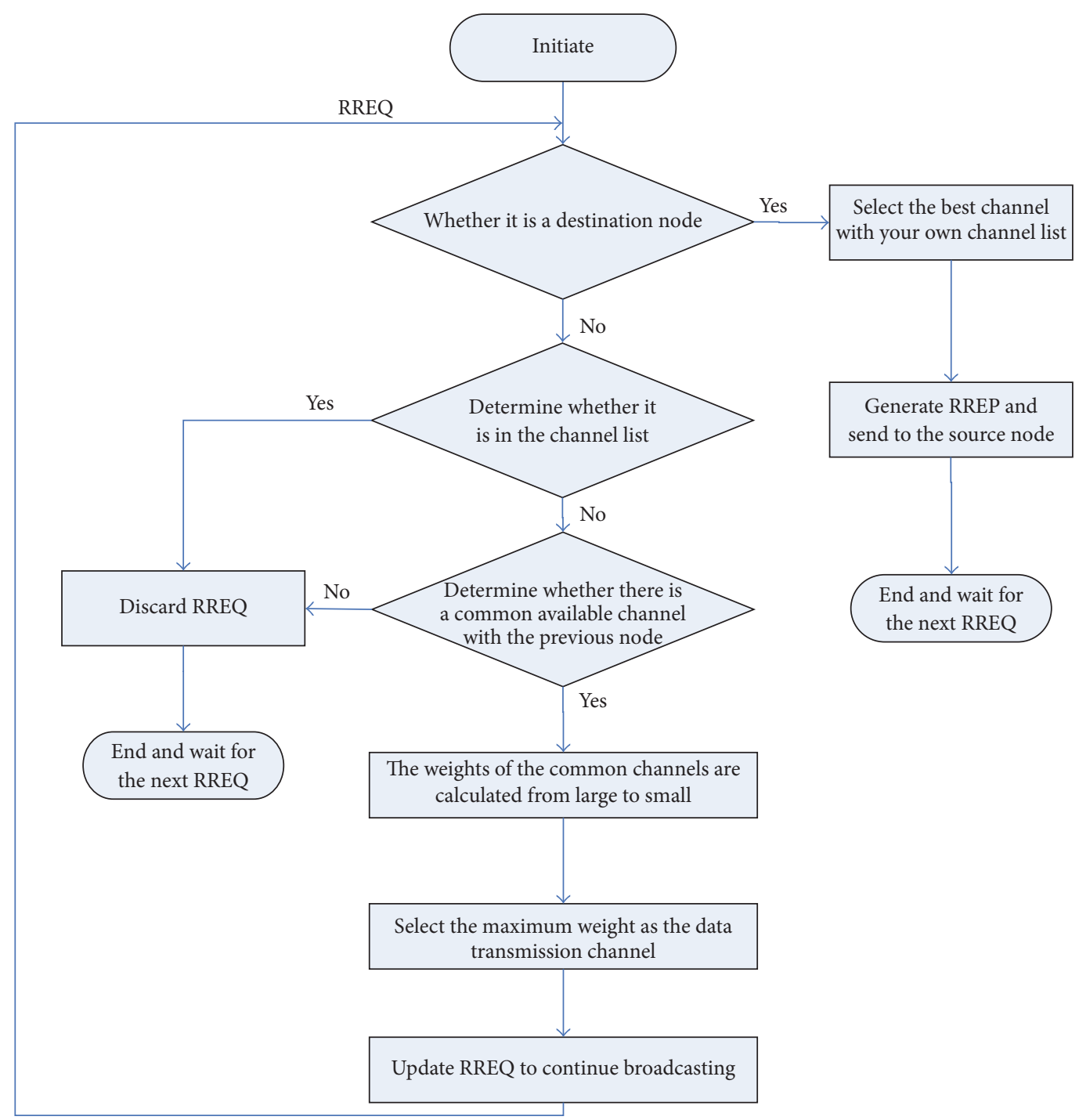

FIGURE 3: Neighbor node processing RREQ control packet.

2.4.1. Route Discovery Phase. After the packet is received by the nondestination node, it will be transmitted again. If source node detects that there is a valid path to destination node, it will send the packet according to the effective path. On the contrary, the route discovery process which is divided into routing request phase and routing response phase will be started by source node to create a new path.

In routing discovery process, route request packet (RREQ) is broadcasted by source node to establish reverse route. And RREQ format is shown in Table 2, including source node address, destination node address, routing list from source to current node (relay node ID in the path, selected channel, and channel availability probability), and channel status table of current node.

The flow chart of neighbor node processing RREQ is shown in Figure 3. After receiving RREQ, neighboring node determines whether it has been received firstly and, if it has been received, it directly discards to prevent routing loops. Otherwise combining with its own channel state table, use
TABLE 2: RREQ format.

\begin{tabular}{l}
\hline RREQ format \\
\hline RREQ_ID \\
TimeStamp \\
Source node ID and address \\
Destination node ID and address \\
The channel state table of the current node \\
Source node to the current node's routing list (relay node ID in the \\
path, selected channel and channel availability probability)
\end{tabular}

the proposed channel selection algorithm to select a suitable channel, and then update its own routing table, and forward RREQ after modifying the corresponding field.

2.4.2. Route Establishment Phase. The route establishment phase is initiated by destination node to select an optimal 
route, according to the received multiple RREQ packets. The route reply packet (RREP) is sent by destination node and the forward route is established. After receiving the RREP packet, source node sends the data according to it. The process is analyzed as follows.

After destination node receives the first RREQ, it starts a timer to switch on route establishment process. If other RREQ are not received after the timer is finished, reverse route is established according to the first RREQ packet. All path information and selected channels are encapsulated in the RREP control packet to send along this path. The source node address, the destination node address, and the routing list of the entire route (relay node ID in the path and channel selection information) are contained in RREP. If the destination node receives multiple RREQ packets, the routes need to be filtered. And the following is the process of algorithm:

(1) Calculate the delivery rate of entire link according to available channel probability in each RREQ packet. Assume that

$$
\text { path }(S, D)=\left\{S, f w_{1}, f w_{2}, \ldots, f w_{i}, \ldots, f w_{h}, D\right\},
$$

where $f w_{i}$ represents $i$ th relay node, and the routing link of $i+1$ hop is only related to $i$ hop, so the whole path is a Markov process, and link delivery rate is calculated as follows:

$$
\begin{aligned}
P_{S, D} & =P\left(S, f w_{1}, f w_{2}, \ldots, f w_{i}, \ldots, f w_{h}, D\right) \\
& =\mathrm{P}\left(L_{S, f w_{1}}=1, L_{f w_{1}, f w_{2}}=1, \ldots, L_{f w_{i}, f w_{i+1}}\right. \\
& \left.=1, \ldots, L_{f w_{h}, D}=1\right)=P\left(L_{S, f w_{1}}=1\right) P\left(L_{f w_{1}, f w_{2}}\right. \\
& =1) \cdots P\left(L_{f w_{h}, D}=1\right),
\end{aligned}
$$

where $L_{f w_{i}, f w_{i+1}}=1$ indicates that the channel can be used by cognitive user. Only the route satisfying constraint $P_{S, D} \geq P$ can be used as a candidate route, and the value of $P$ is related to network environment.

(2) Calculate $D_{\text {RREQ }}$ according to the time recorded by TimeStamp in each RREQ packet and the current time, and sort them from small to large.

(3) Select the path that first satisfies link delivery rate constraint from $D_{\mathrm{RREQ}}$ sequence.

2.4.3. Route Maintenance Phase. The authorization channel may be occupied by PU at any time after route is established, resulting in failure of communication. Therefore, the established route must be maintained. The following algorithm is used for route maintenance:

(1) Local maintenance: firstly, the upstream node in the failed link redetects whether there is another commonly available channel for next-hop node, and if they exist, reselect the channel using the channel selection algorithm to continue to transfer data. Otherwise, a route must be rebuilt.
TABLE 3: Simulation parameter.

\begin{tabular}{lc}
\hline Parameter & Value \\
\hline Simulation scene size & $2000 \mathrm{~m} * 2000 \mathrm{~m}$ \\
Number of primary users & 9 \\
Number of cognitive users & 200 \\
Maximum transmission distance & $200 \mathrm{~m}$ \\
Authorized channel bandwidth & $10 \mathrm{MHz}$ \\
Adjacent channel spacing & $10 \mathrm{MHz}$ \\
Number of packets per group & 1000 \\
Packet size & $1024 \mathrm{Bit}$ \\
Stability constraints & 0.6 \\
\hline
\end{tabular}

(2) Rebuilding a route: route error packet (RRER) is sent by cognitive node where the error occurred to source node, informing that it is invalid. Source node stops sending data immediately after receiving RRER and restarts route discovery process.

\section{Performance Evaluation}

In this section, the CSRP is simulated and we first describe our simulation setup and parameters. Then we analyze the simulation results from end-to-end delay and data delivery rate.

3.1. Simulation Parameter Setting. The cognitive nodes are randomly distributed in rectangular plane region. There are nine data channels and a common control channel in the network, and the channel states of different channels are independent and random. The duration of $O N$ and $O F F$ is subject to exponential distribution [12, 13], which we can design two mutual spaced timers to simulate. The channel availability update factor is $\gamma=0.8, \mu=2, p_{\text {init }}=0.8$ which is measured according to experience. Simulation environment parameter settings are as shown in Table 3.

3.2. Performance Analysis. We compare CSRP with TDRP [5] and PUC-JRCA [3] in terms of end-to-end delay and data delivery rate.

The analytical and simulation results for the end-to-end delay against different communication nodes are given in Figure 4. It is noted that the average delay of three routing algorithms increases as the distance of nodes increases, because the mainly increasing distance leads to the increases of hops and delay and processing delay also increase. It can also be seen that TDRP and CSRP are better than PUC-JRCA when the distance is closer, but TDRP needs to reestablish a route due to resulting lower stability to increased latency and PUC-JRCA do not choose route with the best delay, so its end-to-end delay is relative large. CSRP takes full account of channel availability and routing latency and thus performs best with respect to the other two routing protocols.

As shown in Figure 5 that is data delivery rate against different communication nodes, TDRP relative to the other two protocols decreases rapidly as the distance increases, 


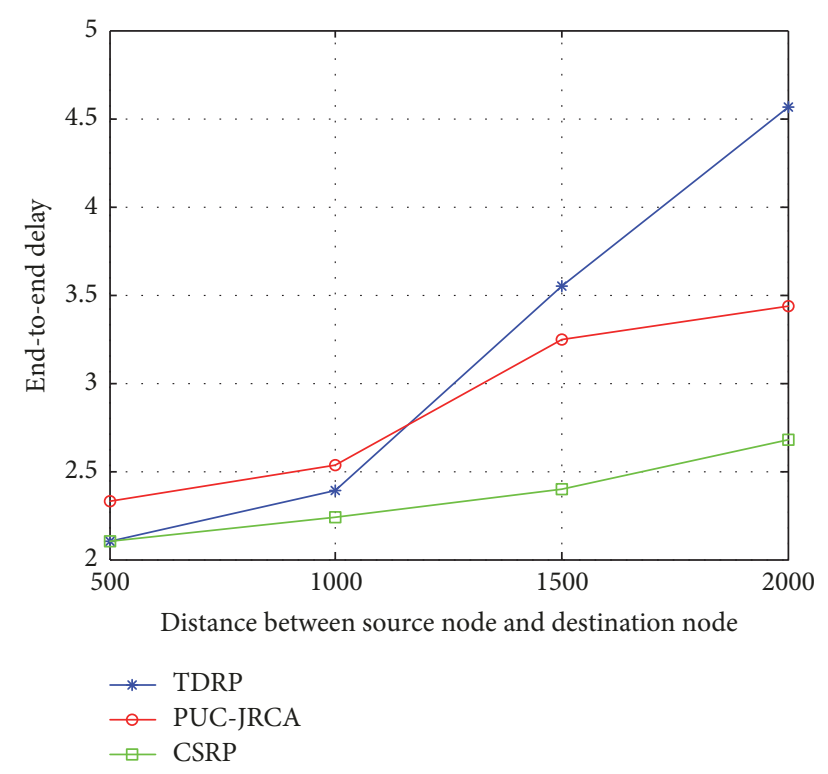

FIGURE 4: End-to-end delay against different communication nodes (packet transmission rate is $20 \mathrm{kbit} / \mathrm{s}$ ).

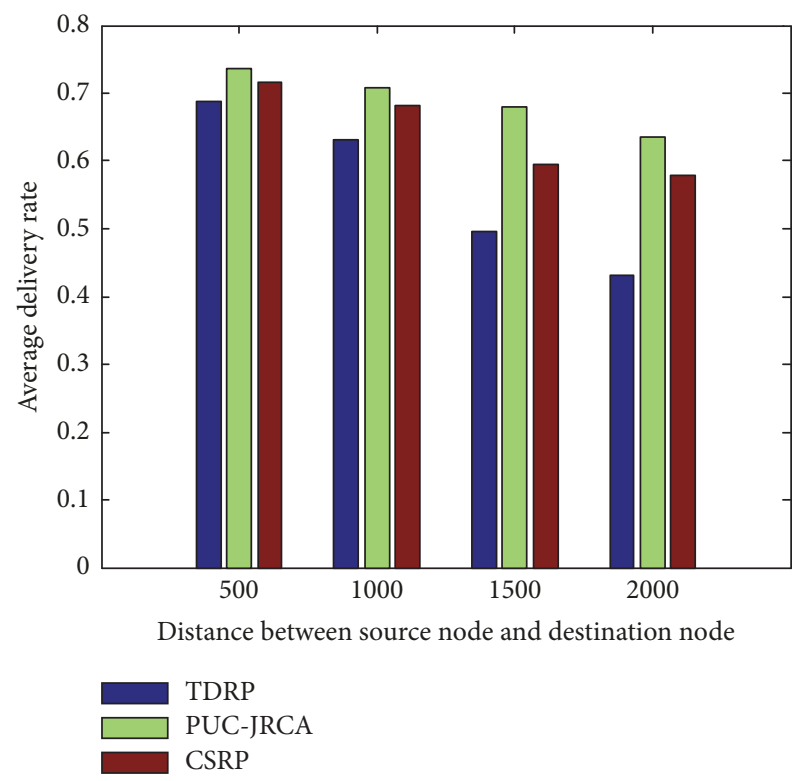

Figure 5: Data delivery rate against different communication nodes (packet transmission rate is $20 \mathrm{kbit} / \mathrm{s}$ ).

because the probability of channel availability is not considered, resulting in a large packet loss rate. However, the channel availability is taken into account in PUC-JRCA and CSRP, so route stability is high and decreases slowly. At the same time, we see that the performance of average delivery rate of PUC-JRCA is better than SARP, because PUC-JRCA only chooses a lower probability of channel availability, but its end-to-end delay performance is poor.

Figure 6 presents end-to-end delay against different packet transmission rate, and it can be seen what end-to-end delay of PUC-JRCA compared with the other two protocol

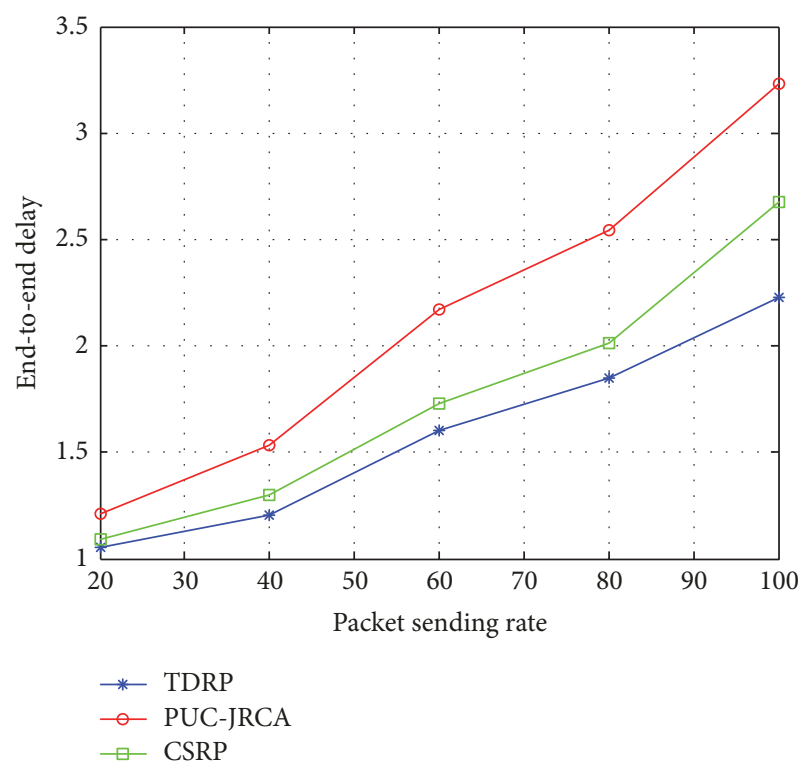

FIGURE 6: End-to-end delay against different packet transmission rate (the distance of communication nodes is $1500 \mathrm{~m}$ ).

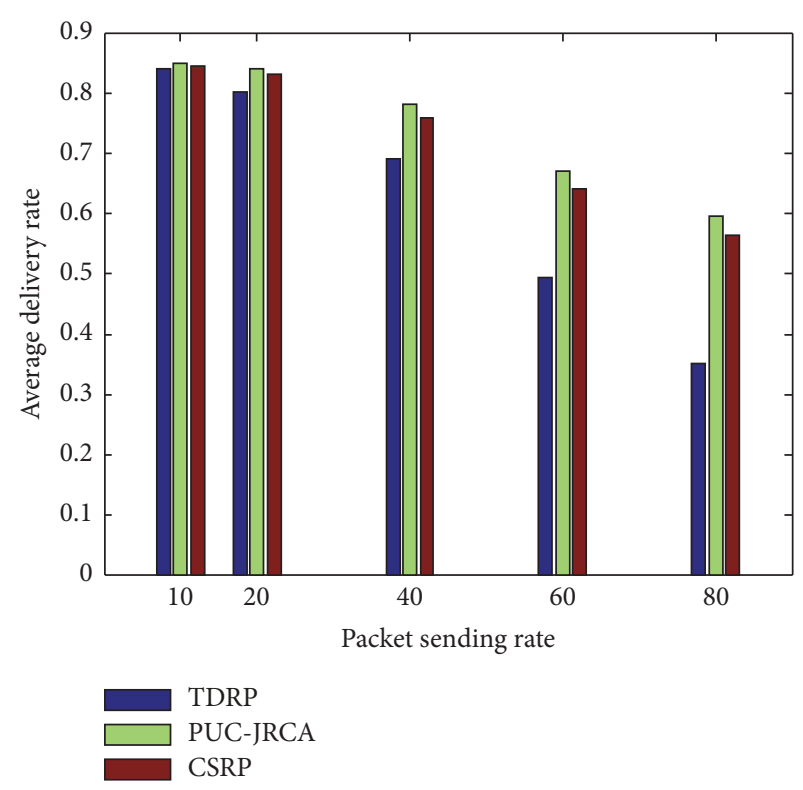

Figure 7: Data delivery rate against different packet transmission rate (the distance of communication nodes is $1500 \mathrm{~m}$ ).

increases faster. Because the selected channel is too single, resulting in that the channel is highly competitive and gets congested, the delay will increase. The channel switching delay is considered in TDRP and CSRP, so it can alleviate the delay problem caused by the increase of transmission rate. At the same time, we see that end-to-end delay of TDRP is better than CSRP. This is because TDRP only selects a low latency channel, but its data delivery rate is very low.

Finally, in Figure 7, we report data delivery rate against different packet transmission rate. And when the packet transmission rate is small, the average delivery rates of three 
protocols are very close. But with the increase of transmission rate, the average delivery of TDRP rate decreased quickly, because the probability of channel availability is not concerned, resulting in the loss of data packets, so the delivery rate decreases. Moreover a larger probability of the route in PUC-JRCA and CSRP is chosen, and the delivery rate is down slowly. Similar to Figure 5, we found that the average delivery rate of CARP is higher than SARP.

Therefore, CSRP considering data delivery rate and endto-end delay are better than the other two protocols, which is higher than TDRP in data delivery rate and shorter than PUC-JRCA in end-to-end delay.

\section{Conclusion}

In this paper, a joint interaction between channel selection algorithm and routing protocol is proposed that can effectively reduce the number of channel failures by considering channel's historical information and routing maintenance to adapt to the environment of multichannel cognitive radio network. The simulation results show that CSRP performs better in packet transmission delay and data delivery rate than TDRP based on delay and PUC-JRCA based on channel availability. In the future work, we will investigate how to adapt metric to optimize the routing performance in dynamic cognitive radio network.

\section{Conflicts of Interest}

The authors declare that they have no conflicts of interest.

\section{Acknowledgments}

This work is supported by the National Key Technology R\&D Program of China (2015BAF32B04-3), the Joint Funds of the National Natural Science Foundation of China (U1404615), the Key Science and Research Program in University of Henan Province (16A460018), the Project of Basic and Advanced Technology Research of Henan Province of China (152300410081), the Natural Science Foundation of Henan Province (162300410098) and the Program for Innovative Research Team (in Science and Technology) in University of Henan Province (15IRTSTHN008), and the National Natural Science Foundation of China $(61671144,61772175)$.

\section{References}

[1] I. F. Akyildiz, W. Lee, M. C. Vuran, and S. Mohanty, "NeXt generation/dynamic spectrum access/cognitive radio wireless networks: a survey," Computer Networks, vol. 50, no. 13, pp. 2127-2159, 2006.

[2] W. Jiang, W. Feng, and Y. Yu, "Spectrum allocation based on auction in overlay cognitive radio network," KSII Transactions on Internet and Information Systems, vol. 9, no. 9, pp. 3312-3334, 2015.

[3] L. Ding, T. Melodia, S. N. Batalama, and J. D. Matyjas, "Distributed resource allocation in cognitive and cooperative ad hoc networks through joint routing, relay selection and spectrum allocation," Computer Networks, vol. 83, pp. 315-331, 2015.
[4] M. Caleffi, I. F. Akyildiz, and L. Paura, "Opera: optimal routing metric for cognitive radio ad hoc networks," IEEE Transactions on Wireless Communications, vol. 11, no. 8, pp. 2884-2894, 2012.

[5] Y. Saleem, K.-L. A. Yau, H. Mohamad, N. Ramli, and M. H. Rehmani, "SMART: A SpectruM-Aware ClusteR-based rouTing scheme for distributed cognitive radio networks," Computer Networks, vol. 91, pp. 196-224, 2015.

[6] K. Habak, M. Abdelatif, H. Hagrass, K. Rizc, and M. Youssef, "A location-aided routing protocol for cognitive radio networks," in Proceedings of the 2013 International Conference on Computing, Networking and Communications, ICNC 2013, pp. 729-733, USA, January 2013.

[7] J. Liu, P. Y. Ren, S. L. Xue, and C. Zhang, "Primary-user behavior based joint routing and channel allocation algorithm in cognitive radio networks," Journal on Communications, vol. 32, no. 11, pp. 183-190, 2011.

[8] G. Cheng, W. Liu, Y. Li, and W. Cheng, "Joint on-demand routing and spectrum assignment in cognitive radio networks," in Proceedings of the IEEE International Conference on Communications (ICC '07), pp. 6499-6503, IEEE, June 2007.

[9] M. Youssef, M. Ibrahim, M. Abdelatif, L. Chen, and A. V. Vasilakos, "Routing metrics of cognitive radio networks: a survey," IEEE Communications Surveys \& Tutorials, vol. 16, no. 1, pp. 92-109, 2014.

[10] S. Jaewoo and R. Srikant, "Improving channel utilization via cooperative spectrum sensing with opportunistic feedback in cognitive radio networks,' IEEE Communications Letters, vol. 19, no. 6, pp. 1065-1068, 2015.

[11] Y. Li, Z. H. Zhang, W. Huang, Y. Wang, and B. Cao, "Multihop routing algorithm with spectrum assignment for cognitive radio networks," Engineering Electronics, vol. 35, no. 4, pp. 852858, 2013.

[12] A. S. Cacciapuoti, M. Caleffi, and L. Paura, "Reactive routing for mobile cognitive radio ad hoc networks," Ad Hoc Networks, vol. 10, no. 5, pp. 803-815, 2012.

[13] S. Bayhan and F. Alagöz, "A Markovian approach for bestfit channel selection in cognitive radio networks," Ad Hoc Networks, vol. 12, no. 1, pp. 165-177, 2014. 


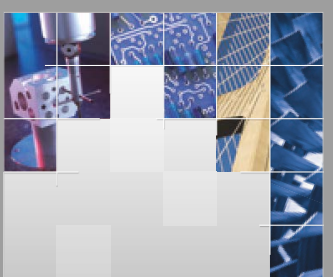

\section{Enfincering}
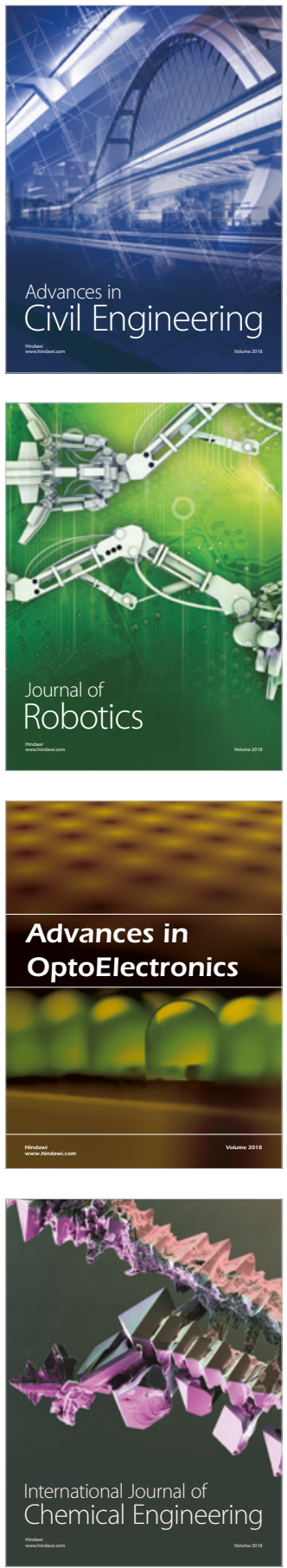

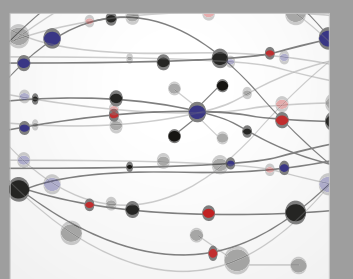

\section{Rotating \\ Machinery}

The Scientific World Journal

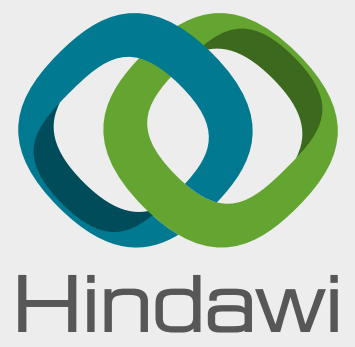

Submit your manuscripts at

www.hindawi.com
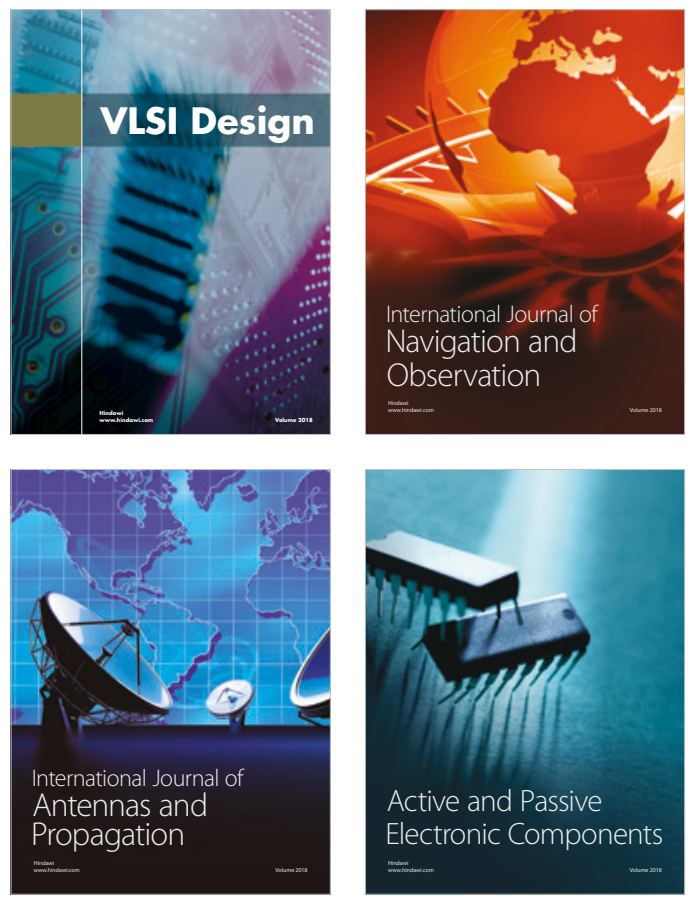
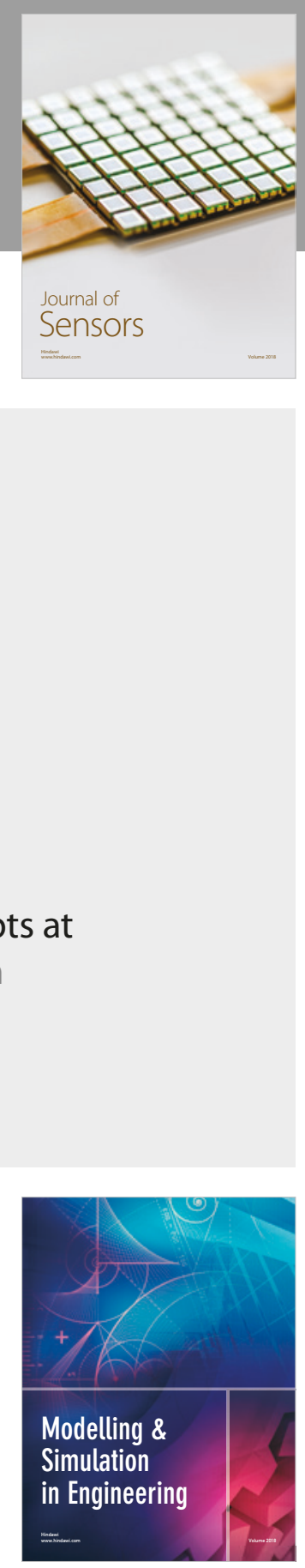

\section{Advances \\ Multimedia}
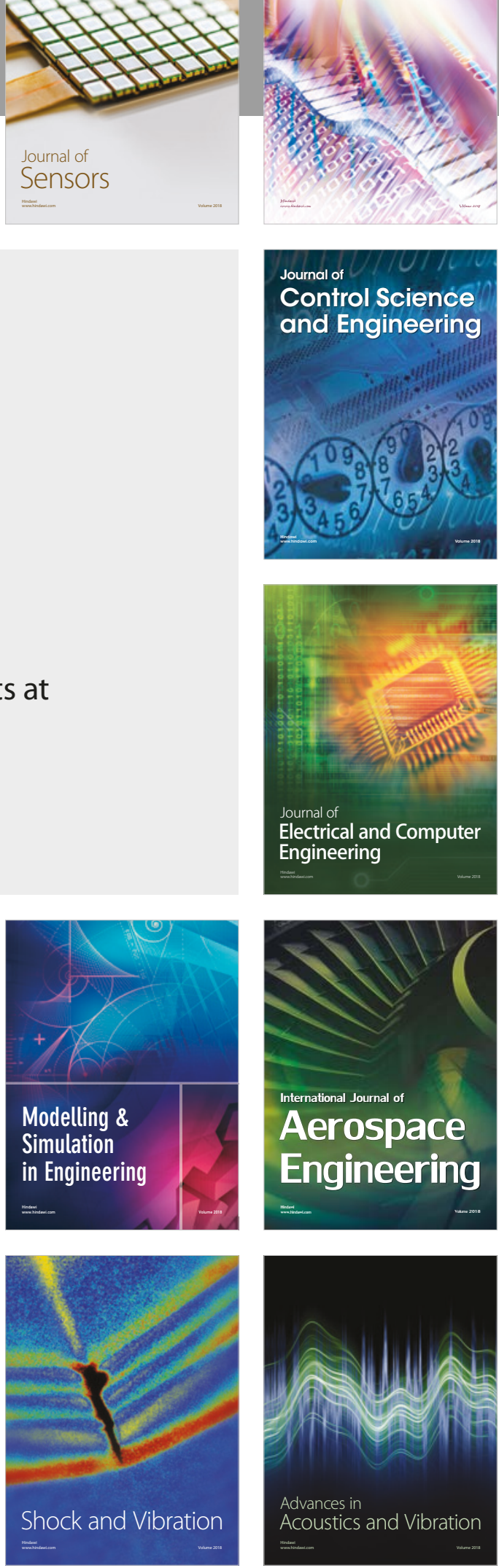